\title{
ORTHOGONAL GASEOUS DISKS IN THE E5 GALAXY IC 4889
}

\author{
J.C. VEGA BELTRÁN \\ TNG, Osservatorio Astronomico di Padova, Italy \\ E.M. CORSINI AND F. BERTOLA \\ Dipartimento di Astronomia, Università di Padova, Italy \\ AND
}

A. PIZZELLA

European Southern Observatory, Santiago, Chile

IC 4889 is an apparently normal E5 elliptical (Jedrzejewsky 1987, MNRAS, 226, 747) with an extended ionized gas emission (Macchetto et al. 1996, $A \& A S, 120,463)$. We performed long-slit spectroscopic observations along different position angles: $0^{\circ}, 10^{\circ}, 90^{\circ}$ and $135^{\circ}$.

Here is a brief description of the observed gaseous and stellar velocity field: (i) an overall counterrotation of the ionized gas with respect to the stars is present along the major axis $\left(P A=0^{\circ}\right)$. Moreover in the inner regions $\left( \pm 10^{\prime \prime}\right)$ both stars and gas reverse their sense of rotation; (ii) both inner gaseous and star counterrotation appear at $P A=0^{\circ}$ and $P A=10^{\circ}$; (iii) the minimum and the maximum velocity gradients for the outer gaseous component are measured at $P A=135^{\circ}$ and at $P A=90^{\circ}$ respectively. The opposite happens for the inner gaseous component; (iv) both inner and outer star components do not show velocity gradient along the minor axis $\left(P A=90^{\circ}\right)$.

The observed gaseous and stellar kinematics can be understood by considering IC 4889 as a triaxial elliptical with a counterrotating stellar core and two orthogonal gaseous structures that have settled onto the two allowed equilibrium planes. The inner gaseous disk rotates in the same plane as that of the stars namely the plane perpendicular to the minor axis, while the outer disk is settled onto the plane perpendicular the major axis. A multiple acquisition event of external material is claimed to explain the presence of such kinematically decoupled structures. 